\title{
Structure of Fermion Nodes and Nodal Cells
}

\author{
Lubos Mitas \\ Department of Physics and CHiPS, North Carolina State University, Raleigh, North Carolina 27695, USA
}

(Received 20 January 2006; published 23 June 2006)

\begin{abstract}
We study nodes of fermionic ground state wave functions. For two dimensions and higher we prove that spin-polarized, noninteracting fermions in a harmonic well have two nodal cells for arbitrary system size. The result extends to noninteracting or mean-field models in other geometries and to Hartree-Fock atomic states. Spin-unpolarized noninteracting states have multiple nodal cells; however, interactions and manybody correlations generally relax the multiple cells to the minimal number of two. With some conditions, this is proved for interacting two and higher dimensions harmonic fermion systems of arbitrary size using the Bardeen-Cooper-Schrieffer variational wave function.
\end{abstract}

DOI: 10.1103/PhysRevLett.96.240402

PACS numbers: 05.30.Fk, 02.70.Ss, 03.65.Ge

A fermion node is a subspace of fermion configurations for which a real wave function describing the fermionic system vanishes due to the antisymmetry. In general, for $N$ spin-polarized fermions in $d$ dimensions the fermion node is a $(d N-1)$-dimensional hypersurface given by an implicit equation $\Psi(R)=0$, where $\Psi$ is the wave function with fermion coordinates $R=\left(\mathbf{r}_{1}, \ldots, \mathbf{r}_{N}\right)$. The location of the nodal manifold is of key importance for quantum Monte Carlo (QMC) methods [1-4] since the exact node enables us to solve the stationary Schrödinger equation with computer time scaling as a low-order polynomial in $N$. Remarkably, even rather crude Hartree-Fock (HF) or post-HF wave function nodes, routinely used in the fixednode approximation QMC methods, provide $\approx 95 \%$ of the correlation energy in a variety of systems with hundreds of valence electrons [1-4]. However, to reach beyond this level of accuracy has proved to be challenging because of a limited understanding of fermion nodes [4-9].

The exact nodes for interacting systems are known only for a very few two-electron triplet atomic states [5,10], and very recently, the exact node of the three-electron ${ }^{4} S\left(p^{3}\right)$ state has been discovered [11]. It turned out that the nodes of these high-symmetry states have rather simple topologies and divide the configuration space into two compact nodal cells in which the wave function is positive or negative ("plus" or "minus" cell). The two nodal cells were found also in noninteracting spin-polarized 2D and 3D fermions with up to 200 particles using a numerical proof [5]. The same noninteracting but spin-unpolarized systems trivially have four nodal cells since the wave function is a product of spin-up and spin-down determinants. Interestingly, an analysis of interacting unpolarized few-particle systems $[6,9,12]$ has revealed that the electron correlation can change the node topologies and number of nodal cells. For example, the correlated wave functions of the $\mathrm{Be}$ atom or $\mathrm{N}_{2}$ molecule exhibit two nodal cells while the corresponding Hartree-Fock ones have four. It was therefore conjectured that the bisection of the configuration space into the two nodal cells might be a generic property of fermionic ground states (with exceptions discussed later). In this Letter we prove that for $2 \mathrm{D}$ spin-polarized noninteracting harmonic fermions the ground state node divides the configuration space into the minimal number of two nodal cells for any system size. The proof method extends to higher dimensions and carries over to other models such as fermions on a sphere, in a periodic box, for atomic states, etc. We show that the same holds, in general, for interacting systems using correlated BardeenCooper-Schrieffer wave functions, and we briefly discuss the implications.

We recall two basic properties of fermion nodes derived by Ceperley [5]. (a) Nondegenerate ground state wave functions satisfy the so-called tiling property, which states that by applying all possible particle permutations to an arbitrary nodal cell one covers the entire configuration space. Note that this does not specify the number of nodal cells. (b) Consider three particles $i, j, k$ in a spin-polarized system with wave function $\Psi(R)$. We call the particles $i, j$, $k$ connected, if there exists a triple exchange $i j k \rightarrow j k i$ path that does not cross the node, i.e., $|\Psi(R)|>0$ along the exchange path. More connected particles, such as the following six ones, can form a cluster: If there exists a point $R_{t}$ such that triple exchanges connect all the particles into a single cluster, then $\Psi(R)$ has only two nodal cells. The tiling property implies that once the particles are connected for $R_{t}$ the same applies to any point in the cell, as further explained in Ref. [5].

We illustrate the properties of nodes in one dimension using spin-polarized fermions in a 1D harmonic oscillator well. The wave function is a Slater determinant $\Psi(1, \ldots, N)=\operatorname{det}\left[\phi_{k}\left(\mathbf{r}_{i}\right)\right]=A \prod_{i} e^{-x_{i}^{2} / 2} \operatorname{det}\{1,2 x, \ldots$, $\left.H_{N-1}(x)\right\}$, where $H_{n}(x)$ is a Hermite polynomial of degree $n$, and $A$ is the normalization. We omit the prefactors and transform the Slater matrix to monomials so that the wave function is given by the Vandermonde determinant

$$
\Psi_{1 \mathrm{D}}(1, \ldots, N)=\operatorname{det}\left\{1, x, x^{2}, \ldots, x^{N-1}\right\}=\prod_{i<j}\left(x_{j}-x_{i}\right) .
$$


The node is encountered whenever two fermions pass through each other and the wave function has $N$ ! nodal cells since any permutation requires at least one node crossing. In general, the derived node is exact for other 1D models, including systems with interactions.

Now consider spin-polarized fermions in a 2D harmonic well. The one-particle states are simply $\phi_{n m}=$ $C_{n m} H_{n}(x) H_{m}(y), n, m=0,1, \ldots$, where $C_{n m}$ includes the Gaussian and normalization which are absorbed into a common prefactor and omitted. The Slater matrix elements can be rearranged to monomials and we write

$$
\Psi_{2 \mathrm{D}}(1, \ldots, N)=\operatorname{det}\left\{1, x, y, \ldots, x^{n} y^{m}, \ldots\right\}
$$

The closed-shell states and the system size are labeled by $M=1,2, \ldots$, where $n+m \leq M$ with the number of fermions given by $N=(M+1)(M+2) / 2$.

Using induction we prove that the wave function in Eq. (2) has only two nodal cells for any $M>0$. This is indeed true for $M=1$ with three-particle wave function $\Psi_{2 \mathrm{D}}(1,2,3)=\operatorname{det}\{1, x, y\}$. In order to show this, it is convenient to extend the particle $2 \mathrm{D}$ coordinates by a "dummy" third dimension as $\mathbf{r}_{i}=\left(x_{i}, y_{i}, 0\right)$. Then $\Psi_{2 \mathrm{D}}(1,2,3)=\mathbf{z}_{0} \cdot\left(\mathbf{r}_{21} \times \mathbf{r}_{31}\right)$, where $\mathbf{z}_{0}$ is the unit vector in the third dimension and $\mathbf{r}_{i j}=\mathbf{r}_{i}-\mathbf{r}_{j}$. Clearly, there are only two nodal cells since the set of vectors, $\mathbf{z}_{0}, \mathbf{r}_{21}, \mathbf{r}_{31}$, is either left or right handed, and the node is encountered whenever the three particles are collinear, i.e., $\mathbf{r}_{21} \times \mathbf{r}_{31}=$ 0 . Also, the particles are connected by triple exchanges without node crossing (e.g., rotate an equilateral triangle).

We now consider a general system with $M>1$ and arrange the particles into a Pascal-like triangle pattern on a rectangular mesh as shown in Fig. 1. For this arrangement the determinant can be explicitly evaluated for any $M$ by subsequent factorization of lines of particles [13]. Because of the space constraints and to simplify the notation, we illustrate the factorization on a few-particle example; the generalization to an arbitrary size is straightforward. For example, for $M=2$ the wave function reads

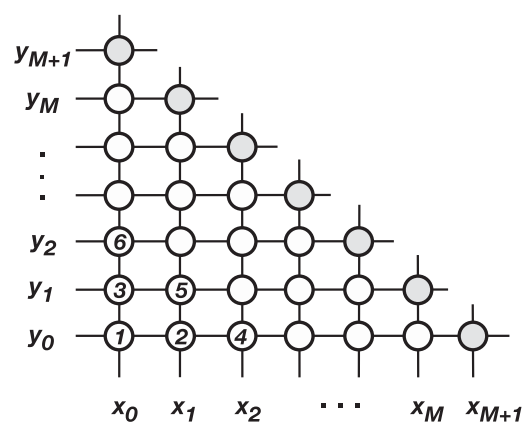

FIG. 1. Particles arranged in a Pascal-like triangle pattern. The circles denote the particle positions and inside the circles are particle labels.

$$
\begin{aligned}
\Psi_{2 \mathrm{D}}(1, \ldots, 6) & =\operatorname{det}\left\{1, x, y, x^{2}, x y, y^{2}\right\} \\
& =\left|\begin{array}{cccccc}
1 & 1 & 1 & 1 & 1 & 1 \\
x_{0} & x_{1} & x_{0} & x_{2} & x_{1} & x_{0} \\
y_{0} & y_{0} & y_{1} & y_{0} & y_{1} & y_{2} \\
x_{0}^{2} & x_{1}^{2} & x_{0}^{2} & x_{2}^{2} & x_{1}^{2} & x_{0}^{2} \\
x_{0} y_{0} & x_{1} y_{0} & x_{0} y_{1} & x_{2} y_{0} & x_{1} y_{1} & x_{0} y_{2} \\
y_{0}^{2} & y_{0}^{2} & y_{1}^{2} & y_{0}^{2} & y_{1}^{2} & y_{2}^{2}
\end{array}\right|,
\end{aligned}
$$

where the particle positions are given in Fig. 1 (our point $R_{t}$ ). Clearly, all the last-row elements containing $y_{0}$ can be eliminated by adding a multiple of the third row. In a similar way we eliminate all the matrix elements containing $y_{0}$, and the determinant factorizes as

$$
\Psi_{2 \mathrm{D}}(1, \ldots, 6)=\prod_{i=1}^{2}\left(y_{i}-y_{0}\right)^{n_{i}} \Psi_{1 \mathrm{D}}(1,2,4) \Psi_{2 \mathrm{D}}(3,5,6),
$$

where $n_{i}$ is the number of particles on line $y=y_{i}$. Note that one of the factors is the $2 \mathrm{D}$ wave function for the system with $M$ reduced by one. The same factorization structure is obtained for an arbitrary $M$ and, obviously, it can be applied recursively. The wave function for a general size $M$ is then given by

$$
\begin{aligned}
\Psi_{2 \mathrm{D}}(1, \ldots, N) & = \pm \prod_{i>0}^{M}\left(y_{i}-y_{0}\right)^{n_{i}} \Psi_{1 \mathrm{D}}\left(I_{0}\right) \Psi_{2 \mathrm{D}}\left(1, \ldots, N / I_{0}\right) \\
& = \pm \prod_{l=0}^{M-1}\left[\Psi_{1 \mathrm{D}}\left(I_{l}\right) \prod_{i>l}^{M}\left(y_{i}-y_{l}\right)^{n_{i}}\right]
\end{aligned}
$$

where $I_{l}=i_{1}^{(l)}, \ldots, i_{M+1-l}^{(l)}$ denotes the labels of particles lying on the line $l$ while $\left(1, \ldots, N / I_{0}\right)$ means that the labels in $I_{0}$ are omitted from $1, \ldots, N$. The sign depends on the number of row exchanges and on the actual ordering of particles. Note the translation invariance of Eq. (5).

For the induction step we assume that triple exchanges connect all the particles in the system of size $M$. Let us show that the same is true for the system of size $M+1$ (see Fig. 1). We first factorize out the line $y=y_{0}$ as given by Eq. (5). One of the factors is the $2 \mathrm{D}$ wave function for the system of size $M$, which contains all additional particles except the one with coordinates $\left[x_{M+1}, y_{0}\right]$. By the original assumption, such a system has all the particles connected. If instead of the horizontal line we factorize out the vertical line $\left(x=x_{0}\right)$, we see that the particle at $\left[x_{M+1}, y_{0}\right]$ is also connected, thus concluding the proof.

The proof can be generalized in several ways. The same arguments apply to three dimensions since particles can be arranged into an appropriate 3D pattern and the wave function evaluated by recursive factorization of planes and lines. In fact, the result holds and the two nodal cell property is correct for arbitrary dimension $d>1$. The proof applies also to other noninteracting or mean-field models with polynomial entries in the Slater matrix such as 
fermions on a sphere or in a periodic box with details given elsewhere [14]. The proof can be modified at least for some open-shell states as well while taking into account node ambiguities if there are degeneracies [5,8].

The proof method can be further combined with symmetries for cases when a complete factorization is not obvious, such as for multishell atomic states. For example, spin-polarized ground states $1 s 2 s 2 p^{n}, n=1-3$ (and beyond), also have two nodal regions. We show this for the ${ }^{6} S\left(1 s 2 s 3 p^{3}\right)$ state in the noninteracting and HF limits. The wave function is written as $\Psi_{\text {at }}(1, \ldots, 5)=$ $\operatorname{det}\left\{\rho_{1 s}^{*}(r), \rho_{2 s}^{*}(r), x, y, z\right\}$, where $\rho_{1 s}^{*}(r)=\rho_{1 s}(r) / \rho_{2 p}(r)$ and $\rho_{2 s}^{*}(r)=\rho_{2 s}(r) / \rho_{2 p}(r)$ since non-negative $\rho_{2 p}(r)$ is factorized out. The dimensionless coordinates are rescaled by the atomic number $Z$ and the Bohr radius $a_{0}$ as $\mathbf{r} \leftarrow$ $Z \mathbf{r} / a_{0}$. Let us place particle 1 at the origin and particles $2-$ 5 on the surface of a sphere with the radius $\eta_{0}$ equal to the radial node of $\rho_{2 s}(r)$ orbital, i.e., $\rho_{2 s}\left(\eta_{0}\right)=0$. For such configurations we obtain

$$
\Psi_{\text {at }}(1, \ldots, 5)=\rho_{1 s}^{*}\left(\eta_{0}\right) \rho_{2 s}^{*}(0) \mathbf{r}_{32} \cdot\left(\mathbf{r}_{42} \times \mathbf{r}_{52}\right)
$$

so that any three-particle exchange from 2, 3, 4, 5 easily avoids node crossing by appropriate positioning and rotations on the sphere. The particle 1 is connected by the exchange $123 \rightarrow 312$ parametrized as $\mathbf{r}_{1}(t)=\eta_{0}[t, 0,0]$, $\mathbf{r}_{2}(t)=\eta_{0}[c(t), s(t), 0]$, and $\mathbf{r}_{3}(t)=\eta_{0}[0,1-t, 0]$, where $t=0(t=1)$ corresponds to the beginning (end) point of the exchange path while $c(t)=\cos (\pi t / 2)$ and $s(t)=$ $\sin (\pi t / 2)$. Setting $\mathbf{r}_{4}=\left[0,0, \eta_{0}\right]$ and $\mathbf{r}_{5}=\left[0,0,-\eta_{0}\right]$, we find that $\Psi_{\text {at }}$ is proportional to

$$
\rho_{2 s}^{*}\left(t \eta_{0}\right) c(t)(1-t)+\rho_{2 s}^{*}\left[(1-t) \eta_{0}\right] s(t) t>0 .
$$

The inequality holds for the whole path $0 \leq t \leq 1$ since $\rho_{2 s}^{*}\left(t \eta_{0}\right)>0$ for $0 \leq t<1$ for both noninteracting and HF cases. The proof can be further extended to more shells such as $1 s 2 s 2 p^{3} 3 s 3 p^{3}$ and $1 s 2 s 2 p^{3} 3 s 3 p^{3} 3 d^{5}$ [14].

Spin-unpolarized systems. - Because of the product of spin-up and -down determinants, the number of nodal cells in noninteracting unpolarized systems is twice the number of cells of the half-filled spin-polarized counterparts. The proof for atomic states above then implies that the HF wave functions for atoms with $Z>3$ up to the third-row elements have four nodal cells.

Interactions in spin-polarized systems. - In general, the shape and topology of the nodal manifold is influenced by interactions and many-body effects. Consider the lowest atomic quartet of $S$ symmetry and even parity ${ }^{4} S^{e}(1 s 2 s 3 s)$ (clearly not the lowest quartet, which is odd ${ }^{4} P^{o}(1 s 2 s 2 p)$ ). The noninteracting wave function is given by $\Psi_{\text {at }}(1,2,3)=\operatorname{det}\left\{\rho_{1 s}(r), \rho_{2 s}(r), \rho_{3 s}(r)\right\}$ and has six nodal cells. Since $\Psi_{\text {at }}(1,2,3)$ depends only on distances, it is quasi-1D and the nodes are the same as the ones given by Eq. (1). For the interacting system the correlation is included by adding to the wave function the lowest (and dominant) double excitation $2 s 3 s \rightarrow 2 p_{x} 3 p_{x}+2 p_{y} 3 p_{y}+$ $2 p_{z} 3 p_{z}$ with a weight $w$. The correlated wave function then allows for exchanges without node crossing. Define $\mathbf{r}_{a}(t)=[0, c(t), s(t)], \quad \mathbf{r}_{b}(t)=[c(t), s(t), 0], \quad \mathbf{r}_{a b}(t)=$ $[1-g(t)] \mathbf{r}_{b}(t)-g(t) \mathbf{r}_{a}(t), \quad \mathbf{r}_{c}(t)=\mathbf{r}_{a b}(t) /\left|\mathbf{r}_{a b}(t)\right|, \quad$ and $\mathbf{r}_{d}(t)=\mathbf{r}_{c}(t) \times \mathbf{r}_{a}(t)$, where $g(t)=3 t(1-t)$. The exchange path $123 \rightarrow 231$ is then $\mathbf{r}_{3}(t)=r_{3}(t) \mathbf{r}_{a}(t), \mathbf{r}_{2}(t)=$ $r_{2}(t) \mathbf{r}_{c}(t)$, and $\mathbf{r}_{1}(t)=r_{1}(t) \mathbf{r}_{d}(t) /\left|\mathbf{r}_{d}(t)\right|$. The radial parts are given by $r_{1}(t)=\eta_{m}+q(2 t-1), r_{2}(t)=\eta_{m}+q(1-$ $t)$, and $r_{3}(t)=\eta_{m}-q t . \eta_{m}$ is the mean value of the radial node of $\rho_{2 s}$ and the first radial node of $\rho_{3 s}$ orbitals while $0<q<a_{0}|w|$ (for the Coulomb $e-e$ interaction $w$ is $\approx-0.05$ ). The path is orchestrated so that in the region where the noninteracting component vanishes the correlation dominates and the particles become connected. This illustrates two points: imposing symmetries at the meanfield level can generate multiple cells and, in general, for $d>1$ the interactions lift this "nodal cell degeneracy" and relax multiple cells to the minimal two.

Interactions in spin-unpolarized systems. - The change from four to two nodal cells due to interactions has been demonstrated for the first time on the Be atom [9] using the two-configuration correlated wave function and the connected cluster construction adapted to spin-unpolarized systems. Consider a simultaneous exchange of an odd number of pairs of spin-up particles and an odd number of pairs of spin-down particles. For noninteracting wave functions such simultaneous pair exchanges imply that the node will be crossed once or multiple times. If there exists a point $R_{f}$ such that during the simultaneous spin-up and -down pair exchanges the inequality $|\Psi|>0$ holds along the whole path, then the wave function has only two nodal cells.

Consider a singlet state of $2 \mathrm{~N}$ particles in a $2 \mathrm{D}$ harmonic well with particles interacting by pair potential. With some restrictions, we show that the correlation included in the Bardeen-Cooper-Schrieffer (BCS) pairing wave function $[7,15]$ given by $\Psi_{\mathrm{BCS}}(1, \ldots, 2 N)=\operatorname{det}[\Phi(i, j)]$ is enough to eliminate the noninteracting four nodal cells and fuse them into the minimal two. Here $\Phi(i, j)=\Phi(j, i)$ is a singlet pair orbital for $i \uparrow$ and $j \downarrow$ fermions and we decompose it into noninteracting and correlated components $\Phi(i, j)=\Phi_{0}(i, j)+\Phi_{\text {corr }}(i, j)$. Using one-particle orbitals we can write $\Phi_{0}(i, j)=\sum_{n+m=0}^{M} \phi_{n m}(i) \phi_{n m}(j)$ while $\Phi_{\text {corr }}(i, j)=\sum_{n+m>M} c_{n m} \phi_{n m}(i) \phi_{n m}(j)$, where $\left\{c_{n m}\right\}$ are variational parameters.

We illustrate this first on a six-particle singlet, $M=1$, $2 N=6$. In cylindric coordinates $(r, \varphi)$ we find $\Phi_{0}(i, j)=$ $1+2 r_{i} r_{j} \cos \varphi_{i j}$, where $\varphi_{i j}=\varphi_{i}-\varphi_{j}$, with the Gaussians and normalization omitted. $\Phi_{\text {corr }}(i, j)$ is constructed as a sum of orbitals from the next unoccupied shell $(n+$ $m=2)$ and we find $\Phi_{\text {corr }}(i, j)=\alpha\left[2\left(r_{i} r_{j} \cos \varphi_{i j}\right)^{2}-r_{i}^{2}-\right.$ $r_{j}^{2}$ ], where a variational parameter $\alpha$ has been included. We place the particles as in Fig. 2(a). For such a configuration $\Psi_{\mathrm{BCS}}(1, \ldots, 6)=8 \alpha r_{a} r_{b} \cos \varphi\left[2\left(r_{a} r_{b} \cos \varphi\right)^{2}-r_{a}^{2}-r_{b}^{2}\right]$. The rotation of the system by $\pi$ exchanges the pair of particles for each spin, and since the wave function is rotationally invariant, it is enough to show that it is nonzero 


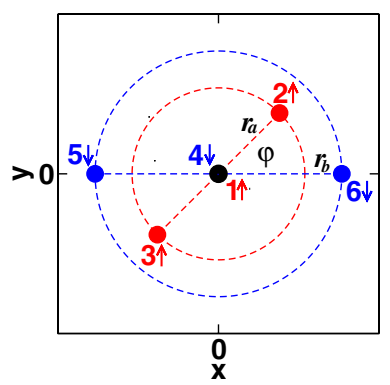

(a)

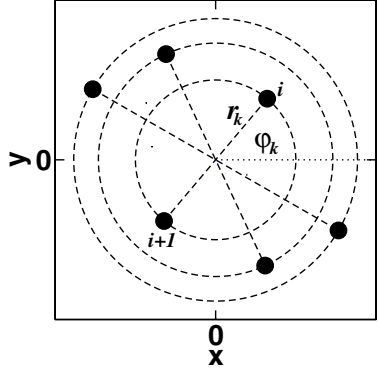

(b)
FIG. 2 (color online). (a) Positions of six 2D harmonic fermions with two particles at the origin and two pairs on circles with radii $r_{a}, r_{b}$. (b) Positions of the spin-up particles for the $M=2$, $2 N=12$ particle singlet. The spin-down positions pattern is similar except for the different radii and angles.

for a single point, e.g., $r_{a}=r_{b}=1, \varphi=\pi / 4$. For $\alpha=0$ the wave function vanishes since the particles lie on the noninteracting node. However, for any interaction which preserves the rotation invariance and gives $\alpha \neq 0$, the BCS wave function has only two nodal cells.

Remarkably, this can be generalized to an arbitrary size. Assume a closed-shell singlet with the total number of particles $2 N=(M+1)(M+2)$, where $N$ is even [for $N$ odd the derivations are the same after placing one particle of each spin to the origin; see Fig. 2(a)]. We form $N_{p}$ pairs of particles $\left(N_{p}\right.$ is odd) in each spin subspace so that, say, the pair $i \uparrow,(i+1) \uparrow$ has coordinates given by $\left(r_{i}, \varphi_{i}\right)=$ $\left(r_{k}, \varphi_{k}\right),\left(r_{i+1}, \varphi_{i+1}\right)=\left(r_{k}, \varphi_{k}+\pi\right)$; see Fig. 2(b). Here $k=1, \ldots, N_{p}$ labels the pairs in the spin-up channel; the spin-down particles are placed similarly and labeled by the pair index $l=N_{p}+1, \ldots, N$. In this configuration the particles lie on the noninteracting node since $\operatorname{det}\left[\Phi_{0}(i, j)\right]=$ $\operatorname{det}\left[\sum_{n+m \leq M} \phi_{n m}(i) \phi_{n m}(j)\right]=\operatorname{det}\left[\phi_{n m}(i)\right] \operatorname{det}\left[\phi_{n m}(j)\right]$ and the rotation of the system by $\pi$ crosses the nodes in both spin channels so that both Slater determinants vanish due to the rotation invariance. Now, if all the $2 N_{p}$ pair distances and angles $r_{k}, r_{l}, \varphi_{k}, \varphi_{l}$ are distinct, then each of the matrices $\left\{\phi_{n m}(i)\right\},\left\{\phi_{n m}(j)\right\}$ has exactly one linearly dependent row; i.e., their ranks are $N-1$. This can be verified directly for small values of $M$ and then using induction for any $M$. Consequently, the matrix $\left[\Phi_{0}(i, j)\right]$ has linear dependence in one row and one column; i.e., it has the rank of $N-1$ as well. In general, adding virtual states through $\Phi_{\text {corr }}(i, j)$ provides independent rows/columns (e.g., $M+1$ independent rows/columns from the first unoccupied shell) which eliminate linear dependency so that $\operatorname{det}\left[\Phi_{0}(i, j)+\Phi_{\text {corr }}(i, j)\right]$ is nonzero. Assuming that the interactions do not break the rotation invariance, the correlated BCS wave functions have only two nodal cells. In fact, this can hold even if the invariance is broken but one would need to show it for the entire exchange path, not only for a single point. The proof extends to $d>2$ and to other models as well [14].
For the classes of fermion systems studied in this work the two nodal cell property indeed appears as a generic feature. That brings up an interesting question: When might this property not apply? We mention just some of the possibilities: (i) additional symmetries and/or boundary conditions can generate additional nodal cells; (ii) nonlocal or very strong/singular interactions can reorder the states (e.g., an excited state becoming the ground state) or significantly change the nodes; (iii) for systems with strong correlations, large degeneracies at the Fermi level, or at quantum phase transitions the properties of nodes are far from clear and require further study. In conclusion, we believe the presented analysis and proofs provide a significant step forward in our understanding of topological properties of fermionic wave functions.

I would like to acknowledge support by NSF Grants No. DMR-0102668, No. DMR-0121361, and No. EAR0530110.

[1] D. M. Ceperley and M. H. Kalos, in Monte Carlo Methods in Statistical Physics, edited by K. Binder (Springer, Berlin, 1979), pp. 145-194; K.E. Schmidt and D. M. Ceperley, in Monte Carlo Methods in Statistical Physics II, edited by K. Binder (Springer, Berlin, 1984), pp. 279355.

[2] B. L. Hammond, W. A. Lester, Jr., and P. J. Reynolds, Monte Carlo Methods in Ab Initio Quantum Chemistry (World Scientific, Singapore, 1994).

[3] M. W. C. Foulkes, L. Mitas, R. J. Needs, and G. Rajagopal, Rev. Mod. Phys. 73, 33 (2001).

[4] J. B. Anderson, J. Chem. Phys. 65, 4121 (1976); J. B. Anderson, Phys. Rev. A 35, 3550 (1987).

[5] D. M. Ceperley, J. Stat. Phys. 63, 1237 (1991).

[6] W. A. Glauser, W. R. Brown, W. A. Lester, D. Bressanini, B. L. Hammond, and M. L. Koszykowski, J. Chem. Phys. 97, 9200 (1992).

[7] M. Casula and S. Sorella, J. Chem. Phys. 119, 6500 (2003).

[8] W. M. C. Foulkes, R. Q. Hood, and R. J. Needs, Phys. Rev. B 60, 4558 (1999).

[9] D. Bressanini, D. M. Ceperley, and P. J. Reynolds, in Recent Advances in Quantum Monte Carlo Methods, II, edited by W. A. Lester, S. M. Rothstein, and S. Tanaka (World Scientific, Singapore, 2002).

[10] D. Bressanini and P. J. Reynolds, Phys. Rev. Lett. 95, 110201 (2005).

[11] M. Bajdich, L. Mitas, G. Drobny, and L. K. Wagner, Phys. Rev. B 72, 075131 (2005).

[12] L. Mitas, G. Drobny, M. Bajdich, and L. K. Wagner, in Condensed Matter Theories, Vol. 20, edited by J. W. Clark (to be published).

[13] C. K. Chui and L. M. Lai, in Nonlinear and Convex Analysis, edited by B.L. Lin and S. Simons (Marcel Dekker, New York, 1987), pp. 23-35.

[14] L. Mitas, cond-mat/0605550.

[15] J. Carlson, S.-Y. Chang, V. R. Pandharipande, and K.E. Schmidt, Phys. Rev. Lett. 91, 050401 (2003). 